\title{
Searching for Spiritual Signatures in SETI Research
}

José G. Funes, S.J. CONICET (National Scientific and Technical Research Council) Universidad Católica de Córdoba, Argentina.

Lucio Florio, Theologian, Universidad Católica Argentina

Marcelo Lares, Astronomer, CONICET-Universidad Nacional de Córdoba.

Mariano Asla, Philosopher, Universidad Austral.

\section{ABSTRACT}

The Search for Extraterrestrial Intelligence (SETI) research assumes that we live in an intelligence-friendly universe. But do we live in a spiritual-friendly universe? We include the spiritual quest in a new multidisciplinary approach to SETI. In a thought experiment, we consider two types of alien civilizations by including a Spiritual factor in the Drake Equation. Using the analogy of planetary biomarkers and the concept of noosphere introduced by Teilhard de Chardin, we propose two spiritual markers that could evidence the presence of a noosphere in an exoplanet.

Keywords: SETI; extraterrestrial intelligence; spiritual; noosphere; civilizations; Drake equation

The discovery of the existence of an extraterrestrial civilization could happen at any time, or perhaps never. Whatever might be the time involved in our search for an extraterrestrial intelligence (ETI), the first contact will, inevitably have a profound impact on our terrestrial worldviews. In the attempt to communicate with a cosmic alter ego, the question who is the other? rises several existential queries. Are we part o fa bigger spiritual cosmic family? Could we establish an intersubjective relationship with other spiritual species? How to prevent the anthropomorphic bias from hindering communication with an ETI? To address these and similar questions in Córdoba, Argentina, we have started project OTHER (acronym for Otros mundos, Tierra, Humanidad, and Espacio Remoto), a multi- disciplinary laboratory of ideas. This work develops more extensively some of the ideas of the White Paper we presented at the Workshop Detecting Extraterrestrial Technology: Signals and Artefacts, organized by the SETI Institute in Mountain View California on March 14-15, 2018. ${ }^{1}$

The Search for Extraterrestrial Intelligence (SETI) program assumes that we live in an intelligence-friendly universe. But do we live in a spiritual-friendly universe? We hardly understand the interaction of life and environment in the evolutionary process of the emergence of our intelligence. For this reason, it is even more difficult to consider the question whether a spiritual genesis is a necessary product of the cosmic evolutionary process or if it could have happened on Earth by chance, as a result of the development of particular events in human history.

To prosecute a research program on extraterrestrial spirit will require the construction of a fertile model. Mary Hesse has observed that models and analogies are integral to scientific practice and advancement. ${ }^{2}$ Analogies and models are particularly necessary in the case of the SETI search, since they aid us in targeting what to look for. ${ }^{3}$ As we 
expand from targeting intelligence alone to spiritual signatures, might the insightful and perhaps prescient achievements of Teilhard de Chardin aid us in model construction?

Teilhard de Chardin's integration of geobiological phenomena with philosophical and spiritual perspectives resulted in ideas such as the emergence of a "sphere of human thinking" (the noosphere) as the next evolutionary stage of the biosphere, and the ultimate expansion of consciousness into the galaxy. Therefore, using the analogy of planetary biomarkers that may reveal the existence of a biosphere, we will focus on the definition and search for spiritual signatures that could evidence the presence of a noosphere in other planets. As SETI is seeking new ways of decoding and coding messages using universal markers, ${ }^{5}$ we propose to include spiritual markers as part of a new approach.

\section{A Working Notion of Spirit}

In our study, the first challenge we face is a working definition of spirit. A complete discussion of this concept exceeds the scope of this paper. We also acknowledge that a working definition depends largely on the model under construction.

Finding a previous definition for spirit can be a strenuous and painful task as evidenced throughout the history of philosophy. Karl Gustzkow, in the Knights of the Spirit, provides a good description of this challenge:

The spirit is a chameleon or one of those delicate fishes of antiquity that, folded and tortured, played in a hundred colors. What is now spirit, ... what must now appear deep to one, superficial to the other, is matter in which there reigns more anarchy ... I describe all our confusion about the spirit. It is found everywhere, in all fields. ${ }^{6}$

The term spirit has been used frequently to translate the Greek words nous and pneuma. The English word "spirit" comes from the Latin spiritus, which, although originally designated "blow," "breath," "exhalation," etc., has also been used to refer to something essentially immaterial and endowed with "reason," like the one designated by the two indicated Greek words. Simplifying the complex discussion that this topic would deserve, for our purpose, we assume that spirit can be described by the concept of nous as something "intellectual" as a "thinking" principle. ${ }^{7}$

As we attempt a multidisciplinary approach to SETI yet still focused on intelligent life, we consider that spiritual beings are those not only capable of processing information-as animals and machines do-but self-conscious subjects, capable of abstract thinking and conceptual language, with ability to transcend the material reality. Thus, the concept of noosphere as introduced by Teilhard de Chardin would be a sphere that hosts living spiritual beings.

Another challenge we face is the choosing of the most appropriate method to study the role of spirituality in the SETI search. We are aware that the issue of the method for studying spiritual reality and its forms has been the subject of the study of many philosophers, though not necessarily scientists. Also, we should discuss whether scientifically defined rationality is the only assumption that could address adequately the spiritual nature of Homo sapiens let alone any extraterrestrials with intelligence. We wish our research program to remain within the methods common to the appropriate natural sciences even if our model is more expansive.

In order to recognize spiritual biomarkers, and knowing that there is no a canonical definition of spirit, we construct what we understand by spirit with a series of features that constitutes, as Ludwig Wittgenstein put it, a sort of "family resemblance" 
(Wittgesntein 1953: § 66-67). We will next look at the analogies going into our model, which will help us recognize an extraterrestrial spiritual signature when we see one.

\section{Spiritual Characteristics of ETI}

As we have said, human nature is our starting point. We could not recognize a spiritual signature or marker without a tacit or explicit comparison with our own specific characteristics. This does not mean that ETI should be identical to ours, but recognizable and therefore to some extent analogous.

In constructing our model of spiritual beings based upon analogies from intellectual thinking as we know it on Earth, we delineate the minimum or necessary traits we expect to find. The following description may be incomplete, but it is an acceptable starting point. Thus, it is expected of a contactable spiritual being:

(1) Self-consciousness.

(2) Symbolic communication. As part of this inter-subjective communication, ET beings should be able to receive and send messages, i.e. capable of developing a SETI-like program.

(3) Aptitude to experience beauty and to communicate the emotions through fine arts, literature, music, etc.

(4) Ability to create and to be convinced by a narrative.

(5) Understanding of the universe in scientific categories, explanation of the universe formulating hypothesis founded on a cause-effect relationship based on experimental data. As Paul Davies ${ }^{8}$ suggests, the emergence of the science could be related to the historic evolution of the Greek philosophy and monotheism. Here there might be a link between spirituality and science.

(6) Capacity to ask why questions transcending the immediate perception of reality, ability to find meaning and values for actions and structures. This ability could allow them to develop philosophical inquiries.

(7) Ability to invent technology making a rational use of natural resources and a potential development of artificial intelligence (AI).

(8) Ability to form social structures at different scales: clans, village-cities, nation-states, global organizations governed by rules-a global consciousness.

(9) Awareness of past, present and future with concern for future generations. Cult of the dead, reverence for ancestors.

(10) Desire of exploration that could imply space exploration. This desire could express and fulfill a cosmic awareness.

(11) Free will, moral sense, altruism, empathy. ${ }^{9}$

(12) Ecological sense. Respectful attitude towards the biosphere and the cosmos.

(13) Ability to develop a personal relationship with the other and a sense of belonging to a spiritual-religious community that can transcend a national or cultural consciousness. The religious predisposition should not be identified with the spiritual nature though religions provide structure and contents for some ways of spirituality. 
There are specific questions that the last characteristic evokes. Would our core spiritual values of love and compassion be a part of an alien religion? Would ET beings even make the same distinction between religion and science; or will it all be mixed together? ${ }^{10}$

If life is a common characteristic of the universe, we can assume that after a long evolutionary process ETI could have evolved into a "Symbolic Species." ${ }^{.11}$ Forms of iconic and indexical communication are present in many species on Earth, but as far as we know, only humans have developed thought and communicate symbolically. ${ }^{12}$ If, to the best of our knowledge, symbolic thinking is strongly correlated with spiritual nature, ${ }^{13}$ then it is fair to say that SETI search for spiritual beings is basically the search for someone with whom we could communicate symbolically at an inter-subjective level.

\section{The Spiritual Factor, Spiritual Markers and the Noosphere}

The Drake equation offers a very helpful educated guess, a rational set of lenses through which to look at probabilities of a potential contact with a technological ET civilization. The equation quantifies the number of civilizations from whom we might receive a radio signal. It has been used as a heuristic device to consider the factors relevant to the search for intelligent life beyond Earth without a strong emphasis on quantifying these terms:

$$
N=R * f_{p} n_{e} f_{i} f_{i} f_{c} L
$$

where, $N$ is the number of civilizations in the Milky Way Galaxy whose electromagnetic emissions are detectable; $R^{*}$ is the rate of formation of stars in our galaxy suitable for the development of intelligent life; $f p$ is the fraction of those stars forming that have planetary systems; $n_{e}$ is the number of planets per solar system with an environment suitable for life; $f_{l}$ is the fraction of suitable planets on which life actually appears; $f_{i}$ is the fraction of inhabited planets on which intelligent life exist; $f_{c}$ is the fraction of intelligent civilizations that develop technology that releases detectable signs of their existence; $L$ is the length of time such civilizations send detectable signs. ${ }^{14}$

One way to approach the search for a spiritual ET is to introduce a spiritual factor or $S$ factor into the Drake equation that could be included in two of the factors of the equation, $f c$ and $L$. Since it is not possible to directly measure the $S$ factor, the strategy that we propose is to search for spiritual signatures or markers.

Until the SETI program detects an unmistakable signal, all speculations about ET intelligence will be nothing but a philosophical thought experiment. ${ }^{15}$

As Mariano Asla points out, a constellation of issues (ontological, ethical and religious) orbits around an anthropomorphic nucleus because any speculation about alternative forms of rationality, always hides a reference to ourselves. ${ }^{16}$

To simplify the complexity of the search, we regroup the thirteen spiritual characteristics described in section 3 into two categories, namely, the relationship of the spiritual ETs to the physical world (S1) and the social relationships in a temporal framework (S2). Thus, the noosphere could be parametrized taking into account these two aspects since a thinker (a scientist or philosopher) can interpret the reality according to physical and social dimensions. We refer to $S 1, S 2$ as spiritual markers.

To carry out a thought experiment we are constrained to follow a process of analogical thinking. Our source is the Earth noosphere and the target is the ET noosphere. ${ }^{17}$ We need 
to identify some elements in the source to transfer them to the target. Here we summarize what a visit from ET would reveal about the uniqueness of humans regarding other animals as described by Laland. ${ }^{18}$ This short description can serve as a source of the analogy:

Imagine an extraterrestrial intelligence studying Earth's biosphere. Which of all the species would it identify as differing from the rest? The answer is humanity. Here are a few reasons:

Population size. Our numbers are out of kilter with global patterns for vertebrate populations. There are several orders of magnitude more humans than expected for a mammal of our size.

Ecological range. Our species distribution is extraordinary. Humans have colonized virtually every region of the terrestrial globe.

Environmental regulation. Humans control vast and diverse flows of energy and matter on unprecedented scales.

Global impact. Human activities threaten and are driving extinct unmatched numbers of species while eliciting strong evolutionary change across the biosphere.

Cognition, communication and intelligence. Experiments demonstrate superior performance by humans across diverse tests of learning and cognition. Human language is infinitely flexible, unlike the communication of other animals.

Knowledge acquisition and sharing. Humans acquire, share and store information on neverbeforeseen scales and build on their pooled cultural knowledge cumulatively from generation to generation.

Technology. Humans invent and mass-produce infinitely more complex and diverse artifacts than other animals.

The extraterrestrials might well be charmed by the elephant's trunk and impressed by the giraffe's neck, but it is humans that they would single out.

In this characterization, what distinctively spiritual markers of our earthling noosphere can we identify? At least the last five items are clearly part of our spiritual features. We regroup these human unique features according to the spiritual markers as it follows:

S1: environmental regulation, global impact, technology.

S2: cognition, communication and intelligence, knowledge acquisition and sharing.

One difference between humans and other animals that we can add to Laland's description is the ability to build big constructions that have civilian and religious goals. This characteristic could be included in S2. For instance, the Great Wall of China, the Saint Peter's Basilica, and the Golden Gate Bridge, are megastructures that serve to different purposes (defensive, religious, communication) and can be detected from space. With our present technology, it would be impossible to detect a megastructure in an exoplanet with a noosphere. However, the recent debate about the nature of the Oumuamua object could change our comprehension and detection of ET civilizations.

This is the first object observed of interstellar origin in the Solar System. Bialy and Loeb consider an artificial origin to account for the observed physical properties. According to them Oumuamua could be a lightsail, floating in interstellar space as a debris from an advanced technological equipment. A more exotic scenario is considered by Bialy and 
Loeb. "Oumuamua may be a fully operational probe sent intentionally to Earth vicinity by an alien civilization."19

Then if we can assume a potential first contact in terms of an electromagnetic message or an encounter with a probe that could carry Al. In the first case, we would be dealing with symbolic communication that will be carried out in a dialogue along years or decades due to stellar distances. In the second case, we will be interacting with unknown technology. Thus, $S 1$ will be the first spiritual marker to be considered. In a second approach, that will require long-period of efforts and studies, $S 2$ will play a more important role.

As Steven Dick points out, assuming that a SETI signal is deciphered and significant information is transmitted, the flow if information between terrestrial civilizations across time finds a tantalizing analogue in the transmission of Greek and Arabic knowledge by way of the Arabs to the Latin West in the twelfth and thirteenth centuries. This is an example of what historian Arnold Toynbee, in his massive Study of History, called "encounters between civilizations in time.".20

We are constructing a heuristic conceptual model based upon terrestrial analogies which will help us recognize an extraterrestrial spiritual signature when we see one.

\section{Two Types of ET Civilizations}

We can continue with our thought experiment and assume that an ET civilization could have developed a system of meanings and values. If this is the case, we would like to find some indicators of the level of spirituality. In an approximate approach, civilizations can be based on a spiritual-moral sense $(S+)$ or the spiritual-moral dimension might be not only missing but might be evolved in an aggressive-predator attitude $(S$-).

In a working hypothesis, from our human source analogue, we assign to $S+$ and $S$ - civilizations some characteristics that we describe below.

$S+$ civilizations could be characterized by:

Respect for other emerging civilizations, openness to other ideas or organizations, willingness to share its cosmic vision and spiritual experience.

Achievement of a certain degree of civilization would require the integration of groups and their differences, maintaining diversity while reaching global agreements. This characteristic implies the respect of individual and minorities rights, and stewardship of the natural resources in their planet.

Desire to seek for other civilizations to deepen their spiritual understanding and to communicate their experience of the sacred and their spiritual-religious cosmic vision.

$S$ - civilizations could be described by:

The pursue of survival at any cost, even when it requires to conquer or enslave another species.

The lack of respect for individual and group liberties, and tendency to dictatorial forms of government

The development of an absolute ego-in opposition to altruism-, and chauvinism-in opposition to openness to diversity - that determines the system of meanings and values. 
These characteristics might be driven by the fear to the otherness and could derive into religious fundamentalism.

We would like to address the following questions. How would the $S$ factor affect the survival or lifespan of a civilization? Would a $S+$ civilization have more chances to survive than a $S$ - civilization? Would a $S+$ civilization be more open to listen and to send messages transferring knowledge, information, and technology? Would a $S+$ civilization have developed a system to listen and decode arriving signals from outer space? On the other side, would a $S$ - civilization be monitoring other civilizations in a lower-level of ET intelligence evolution to control them and exploit their resources?

Since the factors in the Drake equation allow us to explore the abundant of intelligence life in our galaxy, as a first approximation to this wide and complex quest, we propose to simulate the chances of communication among planets where there is a communicating ETI (CETI) in the galaxy; in other words, planets with a noosphere. In our toy model, we consider the Galactic Habitable Zone, the average time between the appearance of CETIs, the average life of a CETI, the average time during which the CETI is active, and the maximum distance at which a CETI is capable of broadcasting or receiving messages.

In a more detailed simulation, we also plan to explore the role of the $S$ factor in a parametrization given by $S 1$ and $S 2$, that could result in $S+$ or $S$-. We also intend to examine if the $S$ factor has any impact on the length of time of CETI civilizations and on the content of the sent message. On the other side, we plan to analyze the influence of the message on the average life of a CETI which receives the message.

To validate our thought experiment, we propose to apply this model to the different civilizations along human history trying to describe different civilizations according to the $S$ factor in its two spiritual markers $S 1$ and $S 2$.

\section{Conclusion: Cosmic Humility}

In this article, we have begun construction of a heuristic model that expands on the concept of intelligence in the SETI research program. With analogies drawn from terrestrial human spirituality, this model should help astrobiologists target spiritual signatures at off-Earth sites. Specifically, Teilhard's notion of the noosphere could benefit the SETI researcher when searching for spiritual biomarkers.

We do not know for sure if we will ever establish contact with a spiritual ET. Nevertheless, the SETI questdrives us to seek abetter understanding of the universe and of ourselves. Inthisendeavor, weneedto putasideourgeocentricandanthropocentricmentality to embrace a more inclusively cosmic worldview. Basically, we need to develop a cosmichumble attitude. What better than the words attributed to Isaac Newton to describe this cosmicmodesty and awareness:"I see myself as achild playing onthe seashore, and from time to time he enjoys discovering a more polished pebble or a more beautiful shell than usual, while before me the immense ocean of truth spreads unexplored."

As Sara Seager, a world expert on exoplanets, has pointed out:

When and if we find that other Earths are common and see that some of them have signs of life, we will at last complete the Copernican revolution-a final conceptual move of the Earth, and humanity, away from the center of the Universe. ${ }^{21}$ 
If this the case, the discovery should have an important impact on the theological reflection. Also, Ted Peters has referred to astrotheology as "the place of cosmic realization within the expression of our faith."22 Then the next Copernican revolution will help astrotheologians to reflect on the divine creation using categories from a cosmic place that is less earthling-centered. This is part of our spiritual journey.

\section{Notes}

1. J.G. Funes et al., "The Spiritual Quest in the SETI Research." (White Paper presented at the Workshop Detecting Extraterrestrial Technology: Signals and Artefact, organized by the SETI Institute in Mountain View California, March 14-15, 2018. https://daiworkshop.seti.org/ sites/default/files/workshop-2018/Funes\%20-\%20The\%20Spiritual\%20Quest\%20in\%20the\% 20SETI\%20Research.pdf (accessed March 5, 2019)).

2. M.B. Hesse, Models and Analogies in Science (Notre Dame: University of Notre Dame Press, 1966).

3. For a discussion of the validity and role of analogues in the treatment of the societal impact of extraterrestrial life and direct contact with intelligence: see S.J. Dick, "The Societal Impact of Extraterrestrial Life: The Relevance of History and the Social Sciences," in Astrobiology, History, and Society, Life Beyond Earth and the Impact of Discovery, ed. D.A. Vakoch (Berlin, Heidelberg: Springer-Verlag, 2013). Kindle edition, 226; and S.J. Dick, Astrobiology, Discovery, and Societal Impact (Cambridge: Cambridge University Press, 2018). Kindle edition, 64 .

4. B. Lehman and M.A. Persinger, "Convergent Quantification and Physical Support for Teilhard de Chardin's Philosophy Concerning the Human Species and Evolutionary Consciousness." Open Journal of Philosophy 5 (2015), 338-350. https://doi.org/10.4236/ojpp.2015. 56042

5. N. Cabrol, "Alien Mindscapes - A Perspective on the Search for Extraterrestrial Intelligence," Astrobiology 16:9 (2016): 661.

6. H. Buchner, "Espíritu," in Conceptos fundamentales de Filosofía, Tomo II, eds. H. Krings, H.M. Baumgarttner, and C. Wild (Barcelona: Herder, 1978), 27.

7. For a detailed introduction see: P. Macdonald, History of the Concept of Mind: Volume 1: Speculations About Soul, Mind and Spiritfrom Homer to Hume. (London, New York: Routledge, 2017).

8. P. Davies, Un silencio inquietante: La nueva búsqueda de inteligencia extraterreste (Spanish Edition, Grupo Planeta, 2011), Kindle edition, location 1482.

9. On ET altruism and ethics in the cosmos see D.A. Vakoch, (ed.), Extraterrestrial Altruism: Evolution and Ethics in the Cosmos (Springer, 2014). Kindle edition.

10. D. Grinspoon, Lonely Planets: The Natural Philosophy of Alien Life (HarperCollins, 2009). Kindle edition, location 414.

11. T. Deacon, The Symbolic Species: The Co-evolution of Language and the Brain (W.W. Norton \& Company, 1997). Kindle edition.

12. T. Schilhab, F. Stjernfelt, and T. Deacon. "Introduction," in The Symbolic Species Evolved (Springer, 2012), iBooks.

13. T. Deacon and T. Cashman, "The Role of Symbolic Capacity in the Origins of Religion," Journal for the Study of Religion. Nature and Culture 3:4 (2009): 26.

14. D.A. Vakoch and M.F. Dowd, eds., The Drake Equation (Cambridge, UK: Cambridge University Press, 2015), xix.

15. M.G. Asla, "Xenophilosophy andthe Knowledge of Ourselves." Science, Religion and Culture 3:2 (2016): 96-109.

16. Ibid.

17.S.J. Dick, "The Societal Impact of Extraterrestrial Life: The Relevance of History and the Social Sciences," in Astrobiology, History, and Society, Life Beyond Earth and the Impact of Discovery, ed. D.A. Vakoch (Berlin, Heidelberg: Springer-Verlag, 2013). Kindle edition, 226. 
18. K. Laland, "How We Became a Different Kind of Animal. An Evolved Uniqueness," Scientific American, September 2018, 33.

19. S. Bialy and A. Loeb, "Could Solar Radiation Pressure Explain ‘Oumuamua's Peculiar Acceleration?," The Astrophysical Journal Letters 868 (2018): L1.

20. S.J. Dick, "The Societal Impact of Extraterrestrial Life: The Relevance of History and the Social Sciences," in Astrobiology, History, and Society, Life Beyond Earth and the Impact of Discovery, ed. D.A. Vakoch (Berlin, Heidelberg: Springer-Verlag, 2013). Kindle edition, 226.

21. S. Seager, "Searches for Habitable Exoplanets," in Frontiers of Astrobiology, eds. C. Impey, J. Lunine and J. Funes (New York: Cambridge University Press, 2012), 23.

22. T. Peters, "Introducing Astrotheology," in Astrotheology: Science and Theology Meet Extraterrestrial Life, ed. T. Peters (Cascade Books, 2018). Kindle edition, Chapter 1, location 493.

José G. Funes S.J., Lucio Florio, Marcelo Lares \& Mariano Asla (2019) Searching for Spiritual Signatures in SETI Research, Theology and Science, 17:3, 373-381, DOI: $10.1080 / 14746700.2019 .1632550$ 